\title{
Acoustic Analysis of Wild Chimpanzee Pant Hoots: Do Kibale Forest Chimpanzees Have an Acoustically Distinct Food Arrival Pant Hoot?
}

\author{
ADAM P. CLARK ${ }^{1}$ AND RICHARD W. WRANGHAM ${ }^{2}$ \\ ${ }^{1}$ Department of Anthropology, University of Michigan, Ann Arbor, Michigan; ${ }^{2}$ Depcrtment of \\ Anthropology, Peabody Museum, Harvard University, Cambridge, Massachusetts
}

\begin{abstract}
Recordings were made of 193 pant hoots given by 6 adult males during ad lib sampling over a 16-month period in 1988 and 1989 . The presence or absence of a let-down phase, and acoustic measures of the let-down and climax phases of the calls, were compared for different call contexts to determine if an acoustically distinct pant hoot was given uniquely upon arrival at fruiting trees. The greatest proportion of pant hoots with a let-down (LD pant hoots) occurred immediately upon arrival at fruiting trees. However, LD pant hoots also occurred at other times during feeding bouts. The frequency and duration of the first exhaled element of 19 letdown phases were measured, and four measures were made on the highest pitched element of 49 climax phases: duration, maximum and minimum frequencies, and average frequency. No differences were found in these acoustic measures that distinguished calls given immediately upon arrival at a food tree from calls given later during feeding bouts. Thus no evidence was found that an acoustically distinct pant hoot was given uniquely upon arrival at fruiting trees. However, the analyses did suggest that identifiable pant hoot variants are given in different social contexts. The proportion of LD pant hoots decreased in more interactive social contexts, and other acoustic features may be available to distinguish this pant hoot variant at long distances. We suggest that different pant hoot variants might broadcast information specific to social, as opposed to ecological, context. 1993 Wiley-Liss, Inc.
\end{abstract}

Key words: chimpanzee, pant hoot, acoustic analysis, semanticity

\section{INTRODUCTION}

Numerous species of birds and primates are known to vocalize upon arrival at rich or otherwise attractive food sources [gallinaceous birds: Collias \& Joos, 1953; Williams et al., 1968; Stokes \& Williams, 1972; Sherry, 1977; Anderson, 1978; Marler et al., 1986a,b; herring gulls: Frings et al., 1955; house sparrows: Elgar, 1986; ravens: Heinrich, 1988; cliff swallows, Brown et al., 1991; spider monkeys:

Received for publication March 12, 1992; revision accepted March 6, 1993.

Address reprint requests to Adam Clark at his present address, 135 Puhalka Road, Newfield, NY 14867.

(C) 1993 Wiley-Liss, Inc. 


\section{0 / Clark and Wrangham}

Klein, 1972, in Chapman \& Lefebvre, 1990; toque macaques: Dittus, 1984; chimpanzees: Wrangham, 1977; Hauser \& Wrangham 1987]. These food calls are known to recruit other individuals, but it is unclear whether the calls provide information about the caller, the food, or some other aspect of the context. Part of the difficulty in interpreting the information content of food calls is that they can be given in a variety of contexts, with food either present or absent. Food calls given in non-food contexts could in theory be used deceptively to attract others to non-existent food. Distinguishing this possibility from non-semantic alternatives is difficult [Marler et al., 1986b]. Therefore the suggestion that chimpanzees have a food call given exclusively in feeding contexts is of special interest.

Wrangham [1977] and Plooij [personal communication in Wrangham, 1977] suggested that the pant hoots given by chimpanzees in Gombe National Park (Tanzania) upon arrival at large food trees constituted an acoustically distinct vocalization, the food-pant-hoot. Pant hoots are loud vocalizations, audible at distances estimated as at least $600 \mathrm{~m}$ [A. Clark, personal observation], $1.6 \mathrm{~km}$ [Reynolds \& Reynolds, 1965], or $2 \mathrm{~km}$ [Ghiglieri, 1984]. They commonly occur as part of extended, long-distance vocal exchanges between individuals out of sight of one another [Goodall, 1965; Reynolds \& Reynolds, 1965; Nishida, 1968; Sabater-Pi, 1979; Ghiglieri, 1984]. The food-pant-hoot seemed to have clear behavioral consequences, because it was associated with an increase in the rate at which other individuals arrived at the food tree [Gombe: Wrangham, 1977; Kibale: Ghiglieri, 1984], thus providing the first indication that a specific chimpanzee vocalization might function semantically. However, no acoustic analyses have been conducted to test the semantic hypothesis. In this paper we combine acoustic and contextual analyses to determine whether an acoustically identifiable variant of the chimpanzee pant hoot vocalization is given uniquely upon arrival at food trees. This analysis constitutes an initial step toward determining if particular chimpanzee vocalizations have specific external referents.

\section{METHODS}

\section{Study Area}

The research reported here was carried out between May, 1988, and December, 1989 , in the Kanyawara study area of the Makerere University Biological Field Station, located in the north-central part of the Kibale Forest Reserve, Toro district, western Uganda. Detailed descriptions of the forest may be found in Kingston [1967], Wing and Buss [1970], Struhsaker [1975], Ghiglieri [1984], and Skorupa [1988]. Descriptions of the study animals and how they were located for observation sessions may be found in Wrangham et al. [1992] and Clark [1993].

\section{Description of the Pant Hoot Vocalization}

The chimpanzee pant hoot is a highly variable vocalization uttered by individuals of all ages and sexes in a wide range of behavioral contexts, including arrival at a new food source, during travel, in response to hearing the calls of another individual or party, during social excitement, and during nesting [Goodall, 1986]. Marler and Hobbett [1975] provided the first detailed acoustic description of the pant hoot from Gombe chimpanzees. Analysis revealed that sufficient acoustic variability was present to discriminate between individuals and sexes. No attempt was made to discriminate between calls by context. Instead, the authors described a "typical pant-hooting sequence," the duration of which ranged from 3-23 seconds. This sequence included four phases, two of which are very similar to calls given in other contexts: 1) the "introduction," often omitted, comprised of one or more long, unmodulated, low-pitched elements, similar to the "hoo" vocalization 




\section{Seconds}

Fig. 1. Sound spectrogram of a four-phase pant hoot vocalization by adult male SY from the Kibale Forest. Arrows point to the first exhaled element of each phase. All sounds in the introduction are exhalations. In this exemplar, the first arched element of the build-up phase is exhalation, followed by 4 inhalation/exhalations, and ending with an inhalation preceding the first element of the climax phase (the roughness of the elements is due to background noise). The climax phase has two exhaled elements (the first beginning right at the start of the phase), with a lower pitched inhalation between them, and another inhalation preceding the let-down. Acoustic analyses of the climax phase used the highest pitched of the exhaled climax elements, which in this case is the second one. The let-down phase here has three exhaled elements, the first starting at the beginning of the phase, with exhalations between the first and second and between the second and third exhaled elements.

given by distressed individuals; 2) the "build-up," comprised of a highly variable number of low-pitched, unmodulated elements, shorter than those in the introduction, but delivered with increasingly higher amplitude; 3) the "climax," generally absent in females, characterized by one or more long, high-pitched, frequency modulated elements, similar to the scream; and 4) the "let-down," often omitted, similar to the build-up. A sound spectrogram of a Kibale pant hoot including all four phases is shown in Figure 1.

\section{Pant Hoot Recordings}

Tape recordings of pant hoots ( $\mathrm{N}=222$ calls) were made by one of us (A.P.C.) in the Kibale Forest using a Marantz PMD430 stereo cassette recorder (one channel only), a Sennheiser directional microphone (K3U power module, ME80 recording head) with a windscreen, and $\mathrm{CrO}_{2}$ tapes. Recordings were made during ad lib sampling [Altmann, 1974] at a distance of $10-30 \mathrm{~m}$, usually from the base of fruiting trees. Calls were used for analysis if the age and sex of the vocalizer were known. Vocalizing individuals were either in direct view ( $N=210$ calls from individuals in food trees; $\mathrm{N}=7$ calls from individuals on the ground) or approaching (within $5 \mathrm{~min}$ before arrival, $\mathrm{N}=2$ calls) or leaving (within 5 min after departure, $\mathrm{N}=3$ calls) a food tree under observation. The sample includes 6 pant hoots from adult females, 193 from adult males, and 23 from subadult males. Of these, 4 of the 6 adult female calls, 113 of the 193 adult male calls, and 17 of the 23 subadult male calls were by individuals of known identity (see Clark [1991] for a complete tabulation of recorded pant hoots by individual and context). 


\section{2 / Clark and Wrangham}

Of the 113 calls by known adult males, 85 were from a single high-ranking male (SY). Other high-ranking males contributed 9 (ST), 3 (TU), and 12 (BF) calls [see Wrangham et al., 1992, for rank determination]. Low-ranking adult males BB and LB contributed 3 calls and 1 call, respectively. Because of the disproportionate number of calls from SY, the sample is not well suited for an analysis of individual variation among adult males in pant hoot acoustic morphology. Thus the following analysis of data, pooled from all adult males, in large part reflects the behavior of this single high-ranking adult male, SY.

The majority of pant hoots recorded at food trees (172 of 215) were given at eight trees of three species (Ficus natalensis, Ficus ovata, and Ficus saussureana). Because of large fruit crops at these trees, visitation by chimpanzees in general, though not by specific individuals, was highly predictable. Observation of the trees was therefore regularly begun before dawn, permitting us to monitor all early morning arrivals. It was also easiest at these trees to isolate and identify vocalizing individuals.

Few calls were collected away from food trees ( $N=13$ calls total; $N=10$ calls for adult males). Because of the incomplete habituation of the study animals and the dense undergrowth, we normally followed travelling chimpanzees at a distance of 20-100 meters. Consequently identification of callers and contexts was difficult.

\section{Acoustic Analysis}

Sonagrams of calls from known individuals were made on a Kay 5500-1 DSP sonagraph to classify calls according to gross differences in acoustic morphology. Occasionally sonagrams also proved useful, in conjunction with listening, for identifying elements from calls of known individuals when a single other pant hooting individual was simultaneously recorded. If more than two individuals of the same age/sex class were recorded (as often occurred with chorusing adult males), it was generally impossible to associate call elements with a specific individual. In these cases only the presence or absence of the let-down phase could be scored, and assigned to an unknown individual of the given age/sex class. Cases in which more than one age/sex class was chorusing, or in which even the final phases of the calls were obscured, were discarded (88 choruses were discarded, and an additional 119 were not recorded at all). There is at present no way to determine whether the exclusion of these calls introduced a bias into the analysis, which would be the case if one pant hoot variant was more likely to elicit a chorus than another, and was therefore systematically undersampled.

The choice of acoustic measures for quantitative analysis of pant hoot elements was based on the observation of variation in the acoustic morphology of call elements during initial visual inspection of sonagrams. Acoustic measurements of call elements were performed digitally on a Dolch $486 / 25$ computer using a signal processing program developed for the analysis of animal vocalizations [Engineering Design, 1990]. The calls were sampled at 20,000 points per second, yielding an effective analysis bandwidth of $8 \mathrm{kHz}$. Measurements of frequency and duration were made from amplitude spectra produced by 51.2-point Fourier transforms (time resolution $=26 \mathrm{msec}$ ). Because of interference from other chimpanzees or background forest noises, not all elements of all calls could be analyzed. Sample sizes consequently vary slightly for the different analyses.

Measurements. All calls in the sample ( $N=222$ calls) were initially scored for the presence or absence of the let-down phase. This procedure was undertaken in response to two clues about the nature of the proposed food arrival pant hoot. First, R.W.W. listened to pant hoot recordings from a single Kibale adult male (SY) in an effort to identify examples of the food-pant-hoot he had earlier described 
[Wrangham, 1977]. This test was done without knowledge of the call contexts. All of the calls identified by R.W.W. had let-down phases, and it was the let-downs themselves that he pointed to as the identifying characteristic of the call. Second, Goodall [1986, p. 134] states that the "arrival pant-hoot,"

ending (probably according to individual idiosyncrasy) with rather deep roarlike sounds or higher screamlike calls, is typically given on arrival at a good food source and upon joining another party.

This description leaves open the possibility that the "deep roarlike sounds," which describe the let-down phase, are associated preferentially with arrival at food sources rather than with joining conspecifics. Since all recorded pant hoots with a let-down phase were given by adult males, only figures for adult males are presented in the results.

Acoustic measurements were made on the let-down $(\mathrm{N}=19)$ and $\operatorname{climax}(\mathrm{N}=$ 49) phases of pant hoots given by SY. Let-down phases were comprised of 1-3 separate exhaled elements. To ensure independence between calls in the analysis, only one element from each let-down was analyzed. We arbitrarily chose the first exhaled element of each let-down phase (some let-downs contained only one element) for analysis. Two measurements were made on this element: duration (msec) and frequency of strongest energy $(\mathrm{Hz})$. Durations were measured directly from waveforms. The frequency of strongest energy in the let-down was then calculated by performing a 1,024-point Fourier transform $(51 \mathrm{msec})$ beginning at the midpoint of the element (frequency resolution $20 \mathrm{~Hz}$ ).

Climax phases were also comprised of a variable number of elements. As above, only one element from each pant hoot climax was analyzed. The element attaining the highest frequency, as assessed by visual inspection, was selected for analysis. This element was selected because it seemed to be the loudest part of the climax, and therefore the best candidate for transmitting a message over a long distance. Four measurements were made on the lowest band of this climax element: duration (as above), maximum and minimum frequencies, and average frequency of strongest energy. The average was calculated because climax elements are relatively long in duration and vary in pitch over time. A cepstral algorithm was employed to compute 100 calculations, each on a 6-msec (128-point) chunk of the signal. Measurements began at the onset of the call element, with successive measurements made at intervals equal to $1 / 100$ th the duration of the signal. The cepstrum was unable to compute values where the acoustic structure was aperiodic, which typically occurred when there was interference in the recording. Average frequency values were discarded if more than 10 (i.e., $>10 \%$ ) of the calculations over the element were missed.

Comparing calls from different contexts. Five contexts were defined for analysis of the calls. Since relatively few calls were recorded in clearly non-food contexts, the analysis was designed to discriminate between calls given immediately upon arrival at fruiting trees and calls given later during feeding bouts (contexts 1 and 2 below). There are insufficient data at present to determine whether structural differences exist between pant hoots associated with broadly different contexts (e.g., feeding versus patrolling).

The five contexts in which pant hoots were given are as follows:

1. Within $5 \mathrm{~min}$ after arrival at a food tree at which an observer is waiting, and in which the vocalizer is observed to feed $(\mathrm{N}=53$ pant hoots recorded); 


\section{4 / Clark and Wrangham}

2. After 5 min after arrival at a food tree at which an observer is waiting, and in which the vocalizer is observed to feed $(\mathrm{N}=121)$;

3. Within 5 min before arrival at a food tree at which an observer is waiting, and in which the vocalizer is observed to feed (these calls from unseen individuals were included if the only individuals to arrive were adult males) $(\mathrm{N}=2)$;

4. Within $5 \mathrm{~min}$ after leaving a food tree at which an observer is located (only adult male departures included) $(\mathrm{N}=3$ );

5 . In view travelling, resting, or socializing on the ground $(N=5)$.

In addition, pant hoots given within $5 \mathrm{~min}$ after arrival at a food tree were further examined according to whether the call was given within $1,2,3,4$, or $5 \mathrm{~min}$ after arrival and according to whether the tree was empty or occupied by other chimpanzees when the arriving vocalizer's party arrived.

Pant hoots were compared to determine whether acoustic features were associated with different contexts. Three comparisons were carried out. First, the percentage of pant hoots containing a let-down was compared between different contexts. Based on Wrangham [1977] and Goodall's [1986] indications, a higher percentage of let-down pant hoots was expected upon arrival at food trees. Since initial feeding bouts at large fruiting trees typically lasted an hour or more, the 5 min cutoff point was arbitrarily chosen to define the relatively brief arrival context. Second, pant hoots from adult male $S Y$ containing a let-down $(N=19)$ but given in different contexts (context $1, N=14$; context $2, N=5$ ) were compared to determine if they differed in other acoustic features. Differences in the let-down element itself would open the possibility that the structure of the let-down, as well as its presence or absence, is important as an acoustic marker for this vocalization. Third, the climax portions of all analyzable SY pant hoots $(N=49)$ were compared by context (context $1, \mathrm{~N}=17$; context $2, \mathrm{~N}=32$ ), and according to whether the pant hoot had a let-down phase, to determine if context-specific differences exist in this portion of the vocalization.

We note that a change in the proportion of times one pant hoot variant is given in a specified context might reflect changes in the production of other variants, rather than (or in addition to) changes in the production of the one under analysis. Thus an ideal analysis would have compared the rates at which different pant hoot variants were given in different contexts. However, it was always the case that some fraction of the pant hoots given by individuals at fruiting trees were not analyzable acoustically, either because they were masked by vocalizations from other individuals, were only partially recorded, or were not recorded at all. Consequently accurate rate data are not available.

\section{Statistical Tests}

$\chi^{2}$ tests were employed to determine whether significant differences existed between contexts in the percentage of pant hoots that had a let-down phase (i.e., to determine whether the proportion of calls with a let-down was independent of context). Based on observations by R.W.W. and Goodall [1986] indicating that let-down pant hoots are given preferentially upon arrival at fruiting trees, as described above, the tests in this analysis were 1-tailed.

One-way ANOVA was used to test for differences between mean values of acoustic measures of calls in different contexts. Where sample sizes were low $(\mathrm{N}<$ 10), non-parametric tests (Mann-Whitney $U$, Kruskal-Wallis) were also performed, and their results reported if they produced a result different from the ANOVA result at the probability level of $P<.05$, which was used to define significance. 
TABLE I. Percentage of Recorded Pant Hoots With a Let-Down Phase Given Before and After Each of 5 Min After Arrival at Food Trees

\begin{tabular}{|c|c|c|c|c|c|c|}
\hline \multirow{2}{*}{$\begin{array}{l}\text { Min } \\
\text { after } \\
\text { arr. }\end{array}$} & \multicolumn{2}{|c|}{$\begin{array}{l}\text { Percentage of all pant } \\
\text { hoots with a let-down }\end{array}$} & \multicolumn{2}{|c|}{$\begin{array}{l}\text { Percentage of pant hoots given } \\
\text { before the minute with let-down }\end{array}$} & \multicolumn{2}{|c|}{$\begin{array}{l}\text { Percentage of pant hoots after } \\
\text { the minute with let-down }\end{array}$} \\
\hline & Before & After & Empty tree & Occupied tree & Empty tree & Occupied \\
\hline 5 & $43.4(53)$ & $10.7(121)^{* * *}$ & $52.9(34)$ & $26.3(19)^{* *}$ & $14.3(77)$ & $4.5(22)$ \\
\hline 4 & $46.9(49)$ & $10.4(125)^{* * *}$ & $58.1(31)$ & $27.8(18)^{* *}$ & $13.8(80)$ & $4.3(23)$ \\
\hline 3 & $50.0(44)$ & $10.8(130)^{* * *}$ & $58.6(29)$ & $33.3(15)^{*}$ & $14.6(82)$ & $3.8(26)$ \\
\hline 2 & $52.8(36)$ & $12.3(138)^{* * *}$ & $62.5(24)$ & $33.3(12)^{* *}$ & $16.1(87)$ & $6.9(29)$ \\
\hline 1 & $60.0(25)$ & $14.1(149)^{* * *}$ & $75.0(16)$ & $33.3(9)^{* *}$ & $17.9(95)$ & $9.4(32)$ \\
\hline
\end{tabular}

${ }^{a}$ Numbers in parentheses represent total number of calls for that sample. Abbreviations for significance levels of 1-tailed $\chi^{2}$ tests of independence: ${ }^{*}, P<.1 ; * *, P<.05 ;{ }^{* * *}, P<.001$.

\section{RESULTS}

\section{Percentage of Recorded Pant Hoots With a Let-Down Phase in Different Contexts}

Of 193 adult male pant hoots, $38(19.7 \%)$ had a let-down. A significantly higher percentage of pant hoots had let-downs in context 1 (within 5 min after arrival at a food tree: 23 of 53, or $43.4 \%$ ) compared with context 2 (after 5 min after arrival to a food tree: 13 of 123 , or $10.6 \%)\left(\chi^{2}=24.5\right.$, $\mathrm{df}=1, P<.001$, 1-tailed). No recorded pant hoots given while approaching (context $3, \mathrm{~N}=2$ calls) or leaving (context $4, \mathrm{~N}=3$ calls) a food tree had a let-down. No recorded pant hoot given in view of the ground (context $5, \mathrm{~N}=5$ calls) had a let-down.

Since the highest percentage of let-downs occurred within $5 \mathrm{~min}$ after arrival at a food tree (43.4\%), this category was examined in more detail. Table I shows the percentage of pant hoots with let-downs given within 1,2,3,4, and 5 min after arrival at all food trees. At each minute closer to arrival at the tree, the percentage of pant hoots with a let-down increased, from $43.4 \%$ at the 5-min cutoff to $60.0 \%$ (N $=25$ calls) at the 1 -min cutoff. At every minute, a greater percentage of pant hoots with a let-down occurred before the minute $\left(\chi^{2}, P<.001\right)$. Table I also shows percentages for arrivals at empty vs. occupied food trees. At 4 of the 5 min analyzed, the percentage of pant hoots with a let-down phase was higher for arrivals at empty trees $\left(\chi^{2}, P<.05\right.$; for the fifth case, $\left.P<.1\right)$. The percentage at empty trees increased from $52.9 \%$ at $5 \mathrm{~min}$ to $75.0 \%$ at $1 \mathrm{~min}(\mathrm{~N}=34$ and 16 calls, respectively), while the percentage at occupied trees increased from 26.3 to $33.3 \%(\mathrm{~N}=$ 19 and 9 calls, respectively).

To summarize, the proportion of recorded pant hoots with a let-down was higher in the first 5 min after arrival at food trees than in other contexts. Within the first $5 \mathrm{~min}$, the proportion was greatest immediately upon entering the trees, and declined each minute thereafter.

\section{Acoustic Analysis: Comparison of Pant Hoots Given Before and After 5 Min After Arrival at a Food Tree}

The let-down element. Let-downs given in context $1(\mathrm{~N}=14)$ did not differ significantly from those given in context $2(\mathrm{~N}=5)$, in either the duration or frequency of the first exhaled component (1-way ANOVA: $P>.1$ for both). The mean duration of all let-downs was $.19 \mathrm{msec}$ ( range $=.05-.34 \mathrm{msec}, \mathrm{SE}=.022$ ). The mean frequency of all let-downs was $309 \mathrm{~Hz}$ (range $=156-527 \mathrm{~Hz}, \mathrm{SE}=$ 21.86).

The climax element. Table II shows values for the duration, average frequency (Average F), maximum frequency (Maximum F), and frequency range ( $F$ 
106 / Clark and Wrangham

TABLE II. Values of Acoustic Measures of the Highest Pitched Climax Element of 49 Pant Hoots Given By SY

\begin{tabular}{lcccccc}
\hline $\begin{array}{l}\text { Acoustic } \\
\text { measure }^{\mathrm{a}}\end{array}$ & Mean & Range & $\begin{array}{c}\text { Standard } \\
\text { error }\end{array}$ & $\begin{array}{c}\text { ANOVA between } \\
\text { contexts 1 and 2 }\end{array}$ & $\begin{array}{c}\text { ANOVA between } \\
\text { LD and no LD }\end{array}$ \\
\hline Duration $(\mathrm{msec})$ & 0.69 & $.29-1.03$ & .019 & $\mathrm{~F}=.008, P=.930$ & $\mathrm{~F}=.050, P=.824$ \\
Average $\mathrm{F}(\mathrm{Hz})$ & 1,068 & $722-1,207$ & 14.03 & $\mathrm{~F}=.264, P=.610$ & $\mathrm{~F}=.070, P=.792$ \\
Maximum $\mathrm{F}(\mathrm{Hz})$ & 1,397 & $1,007-1,592$ & 17.43 & $\mathrm{~F}=1.57, P=.216$ & $\mathrm{~F}=1.82, P=.183$ \\
F Range $(\mathrm{Hz})$ & 691 & $472-895$ & 14.70 & $\mathrm{~F}=.150, P=.226$ & $\mathrm{~F}=9.02, P<.005$ \\
\hline
\end{tabular}

F, frequency.

${ }^{b}$ Context 1 (within $5 \mathrm{~min}$ after arrival at a food tree): calls with let-down, $\mathrm{N}=12$; without let-down, $\mathrm{N}=5$; context 2 (after 5 min after arrival at a food tree): calls with let-down, $\mathrm{N}=5$; without let-down, $\mathrm{N}=27$.

${ }^{c} \mathrm{LD}$, pant hoots with a let-down; no LD, pant hoots without a let-down.

range) of the highest-pitched climax element of 49 pant hoots given by SY. No significant differences were found between contexts 1 and 2 for any acoustic measure (1-factor ANOVA, $P>.1$ for all comparisons).

The climax element compared between let-down and non-let-down pant hoots. The climax element of pant hoots with a let-down had a significantly greater within-call frequency range than pant hoots without a let-down (Table II) (1-factor ANOVA, $\mathrm{F}=9.02, P<.005$ ). No other significant differences were found (1-factor ANOVA, $P>.1$ for all comparisons).

\section{DISCUSSION}

To test the hypothesis that a chimpanzee loud call is given uniquely upon arrival at food trees we focussed on LD pant hoots (i.e., those with a final roar-like phase called the "let-down" by Marler and Hobbett [1975]), which we identified by ear as being a likely candidate. Our data show that LD pant hoots were indeed associated with food arrival contexts. Thus, LD pant hoots were heard only from adult males, who are the only age/sex class to initiate pant hoots on arrival at unoccupied food trees. The percentage of LD pant hoots (in relation to all pant hoots) rose from $19.7 \%$ in all contexts, to $43.4 \%$ within 5 min of arrival at a food tree, to $60.0 \%$ within 1 min after arrival at all food trees, to $75.0 \%$ for calls given within 1 min after arrival at unoccupied food trees. No non-food-context pant hoots had let-downs; however, the sample of non-food-context calls was too small to know if this was generally true ( $\mathrm{N}=10$ calls from an unknown number of individuals). The proportion of LD pant hoots given within a specified minute after arrival was significantly greater at empty trees than at trees already occupied by chimpanzees, for 4 of the $5 \mathrm{~min}$ analyzed (Table II). These data clearly indicate that LD pant hoots were associated both with food trees and with first arrival at them.

This conclusion might suggest that LD pant hoots are calls adapted for longdistance transmission of information about food. However, although we found that LD pant hoots were associated with the food arrival context, they were also given during other periods of the feeding bout. No acoustic differences were found in the let-down or climax phases of pant hoots given at arrival vs. those given later during feeding bouts (although the climax phases of LD and non-LD calls did differ, suggesting the presence of more than one potential information-bearing component in this call). This means that LD pant hoots either conveyed information about something other than arrival at food sources, or were frequently deceptive. Although we cannot distinguish between these possibilities, frequent deception seems unlikely to be a stable strategy in view of the chimpanzee's ability to adjust social favors in relation to the outcome of interactions [de Waal, 1989]. More 
plausibly, LD pant hoots are one of a number of variants that convey information about the social context, possibly in addition to the food arrival context. Thus we found that significantly more LD pant hoots were given upon arrival at unoccupied than at occupied food trees. Taken together with evidence that adult male chimpanzees are intensely social [Goodall, 1986; Wrangham et al., 1992], our observations suggest that LD pant hoots may convey unusual interest in recruiting other individuals rather than information about a specific ecological context.

\section{CONCLUSIONS}

1. A sample of 222 pant hoots recorded in Kibale Forest was analyzed for the presence of a let-down phase (LD pant hoots). The percentage of LD pant hoots was highest immediately upon arrival at unoccupied food trees, but LD pant hoots also occurred at other times during feeding bouts. Further data from definitively nonfood contexts are needed to determine whether let-downs are primarily associated with feeding in general.

2. Acoustic analyses were conducted on 49 pant hoots given by high-ranking adult male SY. The frequency and duration of the first exhaled element of 19 let-downs were measured. Four measures were made on the highest pitched element of 49 climax phases: duration, maximum and minimum frequencies, and average frequency. These acoustic measures were compared between two different contexts - that is, within 5 min after SY arrived at a food tree, and greater than 5 min after SY arrived at a food tree. No differences were found in these acoustic measures that distinguished calls given immediately upon arrival at a food tree (i.e., in the first $5 \mathrm{~min}$ ).

3. LD pant hoots decreased in frequency in more interactive social contexts. A difference in the climax phase was found between LD and non-LD pant hoots, indicating that there are potentially two information-bearing components in the LD pant hoot. The LD pant hoot might broadcast information specific to social, as opposed to ecological, context.

\section{ACKNOWLEDGMENTS}

We thank the government of Uganda, as represented by the Ministry of Planning and Economic Development and the Forestry Department, for permission to work in the Kibale Forest Reserve. Facilities were provided by the Makerere University Biological Field Station in Kibale Forest and the New York Zoological Society. The Department of Zoology, Makerere University, provided assistance throughout the study. Financial support for this field work came from grants awarded to R.W. Wrangham and M.D. Hauser by the following organizations: the National Science Foundation (BNS-8704458), the National Geographic Society (3603-87), and the Leaky Foundation. Funds were also provided by a MacArthur Foundation Fellowship to R.W. Wrangham, and two one-term Rackham Dissertation Fellowships (University of Michigan, 114650 and 114465) to A.P. Clark.

J.C. Mitani helped with acoustic analyses. Invaluable comments on this manuscript were provided by F.B. Livingstone, W.G. Holmes, J.C. Mitani, B.B. Smuts, C.A. Chapman, L.J. Chapman, R. Seyfarth, K.D. Hunt, and two anonymous reviewers. Assistance in the field was provided by G. Isabirye-Basuta, P. Novelli, G. Kagaba, J. Basigara, C. Kiiza, P. Tuhairwe, J. Byarahunga, and C. Muruuli.

\section{REFERENCES}

Altmann, J. Observational study of behavior: Sampling methods. BEHAVIOUR 49: 227-267, 1974.
Anderson, W.L. Vocalizations of the scaled quail. CONDOR 80:49-63, 1978.

Brown, C.H.; Bomberger Brown, M.; Shaffer, 
M.L. Food-sharing signals among socially foraging cliff swallows. ANIMAL BEHAVIOR 42:551-564, 1991.

Chapman, C.A.; Lefebvre, L. Manipulating foraging group size: Spider monkey food calls at fruiting trees. ANIMAL BEHAVIOR 39:891-896, 1990.

Clark, A.P. THE SOCIOECOLOGY OF WILD CHIMPANZEE VOCAL BEHAVIOR IN THE KIBALE FOREST, UGANDA. Ph.D. dissertation, University of Michigan, 1991.

Clark, A.P. Rank differences in the vocal production of Kibale Forest chimpanzees as a function of social context. AMERICAN JOURNAL OF PRIMATOLOGY 31:159179, 1993.

Collias, N.; Joos, M. The spectrographic analysis of sound signals of the domestic fowl. BEHAVIOUR 5:175-187, 1953.

Dittus, W.P.J. Toque macaque food calls: Semantic communication concerning food distribution in the environment. ANIMAL BEHAVIOR 32:470-477, 1984.

Elgar, M.A. House sparrows establish foraging flocks by giving chirrup calls if the resources are divisible. ANIMAL BEHAVIOR 34:169-174, 1986.

Engineering Design. SIGNAL SOFTWARE MANUAL. Belmont, Massachusetts, Engineering Design, 1990.

Frings, H.; Frings, M.; Cox, B.; Peissner, L. Auditory and visual mechanisms in food finding behavior of the herring gull. WILSON BULLETIN 67:155-170, 1955.

Ghiglieri, M. THE CHIMPANZEES OF THE KIBALE FOREST: A FIELD STUDY OF ECOLOGY AND SOCIAL STRUCTURE. New York, Columbia University Press, 1984.

Goodall, J. Chimpanzees of the Gombe Stream Reserve. Pp. 425-473 in PRIMATE BEHAVIOR: FIELD STUDIES OF MONKEYS AND APES. I. DeVore, ed. New York, Holt, Rinehart \& Winston, 1965.

Goodall, J. THE CHIMPANZEES OF GOMBE: PATTERNS OF BEHAVIOR. Cambridge, Massachusetts, and London, Belknap Press of Harvard University Press, 1986.

Hauser, M.D.; Wrangham, R.W. Manipulation of food calls in captive chimpanzees: A preliminary report. FOLIA PRIMATOLOGICA 48:207-210, 1987.

Heinrich, B. Winter foraging at carcasses by three sympatric corvids, with emphasis on recruitment by the raven, Corvus corax. BEHAVIORAL ECOLOGY AND SOCIOBIOLOGY 23:141-156, 1988.

Kingston, B. WORKING PLAN FOR KIBALE AND ITWARA CENTRAL FOR-
EST RESERVES. Entebbe, Uganda, Forest Department, 1967.

Marler, P.; Hobbett, L. Individuality in a long-range vocalization of wild chimpanzees. ZEITSCHRIFT FUR TIERPSYCHOLOGIE 38:97-109, 1975.

Marler, P.; Dufty, A.; Pickert, R. Vocal communication in the domestic chicken: I. Does a sender communicate information about the quality of food to a receiver? ANIMAL BEHAVIOR 34:188-193, 1986a.

Marler, P.; Dufty, A.; Pickert, R. Vocal communication in the domestic chicken: II. Is a sender sensitive to the presence and nature of a receiver? ANIMAL BEHAVIOR 34: 194-198, $1986 \mathrm{~b}$.

Nishida, T. The social group of wild chimpanzees in the Mahali Mountains. PRIMATES 9:167-224, 1968.

Reynolds, V.; Reynolds, F. Chimpanzees of the Budongo Forest. Pp. 368-424 in PRIMATE BEHAVIOR: FIELD STUDIES OF MONKEYS AND APES. I. DeVore, ed. New York, Holt, Rinehart \& Winston, 1965.

Sabater-Pi, J. Feeding behaviour and diet of chimpanzees (Pan troglodytes troglodytes) in the Okorobiko Mountains of Rio Muni (West Africa). ZEITSCHRIFT FUR TIERPSYCHOLOGIE 50:265-281, 1979.

Sherry, D.F. Parental food-calling and the role of the young in the Burmese red jungle fowl (Gallus gallus spadiceus). ANIMAL BEHAVIOR 25:594-601, 1977.

Skorupa, J.P. THE EFFECTS OF SELECTIVE TIMBER HARVESTING ON RAINFOREST PRIMATES IN KIBALE FOREST, UGANDA. Ph.D. dissertation, University of California, Davis, 1988

Stokes, A.W.; Williams, H.W. Courtship feeding calls in gallinaceous birds. AUK 89:177-180, 1972 .

Struhsaker, T.T. THE RED COLOBUS MONKEY. Chicago, University of Chicago Press, 1975.

de Waal, F.B.M. Food sharing and reciprocal obligations among chimpanzees. JOURNAL OF HUMAN EVOLUTION 18:433459, 1989.

Williams, H.W.; Stokes, A.W.; Wallen, J.C. The food call and display of the bobwhite quail (Colinus virginianus). AUK 85:464476, 1968.

Wing, L.D.; Buss, I.O. ELEPHANTS AND FORESTS. Wildlife Management No. 19. Washington, DC, The Wildlife Society, 1970.

Wrangham, R.W. Feeding behaviour of chimpanzees in Gombe National Park, Tanzania. Pp. 503-538 in PRIMATE ECOLOGY. T.H. Clutton-Brock, ed. New York, Academic Press, 1977.

Wrangham, R.W.; Clark, A.P.; Isabirye-Ba- 
Chimpanzee Food Arrival Pant Hoots / 109

suta, G. Female social relationships and social organization of Kibale Forest chimpanzees. Pp. 81-98 in TOPICS IN PRIMATOLOGY. VOL. 1: HUMAN ORI-
GINS. T. Nishida, W.C. McGrew, P. Marler, M. Pickford, F.B.M. de Waal, eds. Tokyo, University of Tokyo Press, 1992. 